\title{
Multilinguales
}

\section{Apprentissage de la lecture en français au cycle primaire. Analyse du manuel scolaire à la lumière de la psychologie cognitive}

Learning of Reading at The Primary School. Critical Analysis of the School Textbook in the Light of Cognitive Psychology

\section{Atfa Memaï et Abla Rouag}

\section{OpenEdition \\ Journals}

Édition électronique

URL : http://journals.openedition.org/multilinguales/896

DOI : 10.4000/multilinguales.896

ISSN : 2335-1853

\section{Éditeur}

Université Abderrahmane Mira - Bejaia

\section{Référence électronique}

Atfa Memaï et Abla Rouag, «Apprentissage de la lecture en français au cycle primaire. Analyse du manuel scolaire à la lumière de la psychologie cognitive », Multilinguales [En ligne], 6 | 2015, mis en ligne le 01 décembre 2015, consulté le 17 septembre 2019. URL : http://journals.openedition.org/ multilinguales/896; DOI : 10.4000/multilinguales.896

Ce document a été généré automatiquement le 17 septembre 2019

\section{cc)}

Multilinguales est mise à disposition selon les termes de la Licence Creative Commons Attribution Pas d'Utilisation Commerciale - Pas de Modification 4.0 International 


\title{
Apprentissage de la lecture en français au cycle primaire. Analyse du manuel scolaire à la lumière de la psychologie cognitive
}

\author{
Learning of Reading at The Primary School. Critical Analysis of the School \\ Textbook in the Light of Cognitive Psychology
}

Atfa Memaï et Abla Rouag

1 Parmi les objectifs fondamentaux qui se dégagent de la réforme du système éducatif algérien, issus des travaux et des conclusions de la Commission Nationale de Réforme du Système Educatif (2000), et des mesures du gouvernement (2003) ${ }^{1}$, est retenue la revalorisation de l'enseignement des langues étrangères, notamment de la langue française.

En effet, des changements d'ordre qualitatif aussi bien que quantitatif sont introduits dans l'enseignement de cette matière: rénovation des programmes, refonte de la pédagogie, amélioration du matériel didactique, et accroissement du volume horaire (Ferhani, $2006: 11$ 18).

2 Depuis la réforme, l'enseignement de la langue française est avancé d'une année dans les programmes. Celle-ci est enseignée comme première langue étrangère dès la $3^{\text {ème }}$ année du cycle primaire.

Aussi, une nouvelle génération de manuels scolaires est apparue pour accompagner cette réforme car, ceux en usage étaient « considérés par tous comme trop livresques et ne répondant pas adéquatement aux exigences de développement aussi bien des élèves apprenants que de la société » (Tawil, 2005 : 34).

Ces nouveaux manuels, comme les cahiers d'activités, et les documents qui les accompagnent, reflètent une nouvelle approche pédagogique qui place l'élève au centre du processus d'enseignement-apprentissage : il est actif et participe à la construction du savoir (Adel, 2005). 
3 La préoccupation de cette étude est d'apprécier la démarche pédagogique préconisée par cette réforme pour la lecture en français langue étrangère ; la question étant celle de savoir si son enseignement tel qu'il est élaboré dans le nouveau manuel scolaire de 3 ème année est en phase avec le développement actuel des sciences cognitives de l'éducation.

\section{L'apprentissage de la lecture en langue étrangère}

4 La lecture est une activité cognitive complexe qui requiert l'interaction des processus d'identification et de compréhension des mots écrits, c'est-à-dire entre l'identification des mots contenus dans le texte ou processus de bas niveau, et leur interprétation, ou processus de haut niveau (Denhière, 1988 et Carrell, 1990, cités dans Joulia, 2006 : 79 81).

5 L'apprentissage de cette activité consiste principalement en l'acquisition du principe alphabétique qui définit le fonctionnement du code écrit, c'est-à-dire, des mécanismes qui relient les unités graphiques aux unités phoniques (Bentolila, 2006), mais le décodage à lui seul ne suffit pas, et sans accès au sens, on ne peut parler de lecture. En effet, Le décodage de l'écrit, et l'élaboration du sens, sont deux compétences indispensables, une défaillance dans l'une ou l'autre de ces procédures entrave l'accès au savoir-lire.

6 Chez le lecteur expert, les procédures d'identification sont développées et automatisées, donc la reconnaissance des mots et la compréhension se font simultanément; tandis que pour le lecteur novice ou l'apprenti lecteur, les procédures d'identification n'étant pas automatisées, les traitements conduisant à la compréhension sont ralentis parce qu'il focalise toute son attention sur le décodage de l'écrit. L'automatisation du décodage permet de libérer des ressources cognitives et attentionnelles qui seront dirigées vers la compréhension, c'est pourquoi les chercheurs (McLaughlin, 1990, Demont et Gombert, 2004 ; Bonnefoy et Rey. 2008; Dehaene et al., 2011) insistent sur l'automatisation du décodage :

Plus que la maîtrise des traitements graphophonologiques et orthographiques nécessaires à la reconnaissance des mots, c'est leur degré d'automatisme qui constitue un déterminant majeur de la réussite dans l'efficacité de la lecture. De fait, le coût attentionnel de la reconnaissance des mots doit être faible afin que le lecteur puisse consacrer de l'attention aux processus de plus haut niveau impliqués dans la compréhension. (Demont et Gombert, $2004: 246$ 247)

7 La priorité de l'enseignement systématique des correspondances grapho-phonémiques dans l'apprentissage de la lecture se confirme depuis deux décennies, en rapport avec la recherche scientifique sur le cerveau et la lecture qui a connu un bond significatif. Grâce notamment aux nouvelles techniques d'imagerie cérébrale, les chercheurs sont parvenus à maîtriser « la manière dont le cerveau humain reconnaît l'écriture et se modifie au fil de cet apprentissage » (Dehaene et al., 2011 : 9), et à déterminer le rôle essentiel de la région occipito-temporale ventrale gauche dans la reconnaissance visuelle des mots écrits :

In 2000, using functional magnetic resonance imaging (FMRI) and event-related potentials in normal subjects and in two patients with colossal lesions, we found that reading was associated with the activation of a precise and reproducible site in the left lateral occipitotemporal sulcus.....We tentatively termed this region the visual word form area (VWFA). (Dehaene \& Cohen, 2011:254) ${ }^{2}$ 
Pour confirmer l'importance stratégique de cette région du cerveau dans le déroulement des processus cognitifs de la lecture évoqués plus haut, Stanislas Dehaene cite des auteurs tels que Yoncheva, Blau, Maurer \& Mc Candliss (2010, dans Dehaene et al.., $2011: 42-43)$ qui se sont penchés sur l'effet des méthodes syllabiques et globales d'enseignement de la lecture sur le cerveau.

Les résultats de leur recherche ont révélé que chez les sujets soumis à la méthode syllabique, il y avait une plus grande latéralisation à gauche des activations cérébrales, tandis que chez ceux soumis à la méthode globale, il y avait une plus grande latéralisation à droite, région diamétralement opposée à celle apte à visualiser les mots écrits (région occipito- temporale ventrale gauche évoquée plus haut) :

Lorsqu'une personne apprend un alphabet nouveau, l'apprentissage varie massivement selon qu'elle distribue son attention de façon globale ou focale. Si on lui explique que les mots sont constitués de lettres qui représentent des fragments élémentaires du langage parlé, elle apprend rapidement à lire, et l'imagerie cérébrale montre une activation normale de l'aire de la forme visuelle des mots - autrement dit elle devient très vite un décrypteur expert. Si, par contre, on lui présente les mêmes mots comme des formes globales, sans lui dire qu'ils sont composés de lettres, elle n'apprend guère, car sa mémoire est vite dépassée. Surtout, elle active une aire cérébrale inadéquate dans l'hémisphère droit.

La conclusion qui en est tirée quant aux méthodes de lecture à adopter est claire :

En adressant les informations vers ce circuit inapproprié, la stratégie d'attention globale interdit tout apprentissage efficace. Abandonner la lecture globale et prêter attention aux composants élémentaires des mots, un par un, dans un ordre bien précis, est une étape essentielle de l'apprentissage.(Dehaene et al.., op. cit. : 43)

Ainsi, l'efficacité des approches synthétiques pour l'enseignement de la lecture est mise à mal, et celle des approches analytiques est mise en valeur. Avec son équipe de recherche, Stanislas Dehaene $(2010,2011)$, a établi une liste de sept (7) principes pour l'enseignement-apprentissage de la lecture (voir détail en annexe) :

- Principe d'enseignement explicite du code alphabétique

- Principe de progression rationnelle

- Principe d'apprentissage actif associant lecture et écriture

- Principe de transfert de l'explicite vers l'implicite

- Principe de choix rationnel des exemples et des exercices

- Principe d'engagement actif, d'attention et de plaisir

- Principe d'adaptation au niveau de l'enfant

Ces travaux sont d'autant plus importants qu'ils font partie des références pour la mise en place de méthodes d'apprentissage de la lecture en langues étrangères. En effet, comparativement aux travaux de recherche consacrés à l'apprentissage de la lecture en langue maternelle, il en existe peu sur cette activité pour la langue seconde ou étrangère. Gaonac'h $(2000: 5)$ tente de trouver une explication :

(...) L'apprenant d'une langue seconde (...) a de manière générale acquis la maîtrise de la lecture dans sa langue maternelle, de telle sorte que ce qui est propre à la lecture l'utilisation d'un code graphique pour représenter des éléments de langage - ne devrait guère poser de problème (...). C'est sans doute en se référant - explicitement ou implicitement - à ce raisonnement qu'il n'est pas toujours apparu utile de s'intéresser de manière spécifique à la lecture en langue seconde.

Ses recherches (Gaonac'h, 1990, 2000) ont montré les limites de ce « raisonnement » et identifient la nature spécifique des difficultés rencontrées dans l'apprentissage de la lecture en langue étrangère et synthétisées dans les points suivants (1990 : 41-49) ${ }^{3}$ :

- le faible degré d'automatisation des processus de base en langue étrangère conduit le sujet à leur accorder une attention plus importante que ce n'est le cas en langue maternelle; ce qui 
induit un coût cognitif élevé, et ne permet pas de libérer les ressources cognitives et attentionnelles pour le traitement des processus de haut niveau;

- le recours au contexte et aux diverses stratégies compensatoires est gêné ou ralenti, du fait du ralentissement général de la construction de la signification; ce qui rend difficile la reconnaissance globale des mots ;

- le code phonologique est souvent peu disponible, les connaissances orales étant souvent minimes, d'autant plus que dans l'apprentissage d'une langue étrangère l'utilisation de l'écrit précède la bonne maîtrise de la langue orale.

\section{Analyse du manuel de français : méthodologie}

Parmi les méthodes de recueil des données, la grille d'analyse est l'outil qui est le plus utilisé dans l'étude des manuels scolaires. Il est pertinent pour l'analyse de contenu et permet de structurer et d'interpréter la collecte des données. Selon Leray (2008:22), il s'agit d'" un court document récapitulant l'ensemble des éléments de contenu que l'on désire retracer dans le corpus retenu pour étude ».

Nous avons élaboré notre grille de recherche qui comprend dix (10) items énoncés sous forme de questions dont les réponses sont réparties sur une échelle de type Likert ${ }^{4}$, avec quatre (4) possibilités de réponse :

Tableau 1 : la grille d'analyse et objectifs visés

\begin{tabular}{|c|c|c|}
\hline Numéro & Question & Objectifs visés \\
\hline Q- 1 & $\begin{array}{l}\text { Pour l'enseignement de l'alphabet, } \\
\text { est-ce la méthode syllabique, dite } \\
\text { alphabétique, ou traditionnelle qui } \\
\text { est utilisée? }\end{array}$ & $\begin{array}{l}\text { Identifier la méthode d'apprentissage de la } \\
\text { lecture adoptée par le manuel : est-ce la méthode } \\
\text { synthétique? Ou analytique? }\end{array}$ \\
\hline Q- 2 & $\begin{array}{l}\text { L'alphabet complet et en ordre, est- il } \\
\text { exposé de manière pertinente à } \\
\text { l'élève, de telle sorte qu'il puisse } \\
\text { connaître l'ordre alphabétique? }\end{array}$ & $\begin{array}{l}\text { Estimer le caractère explicite des contenus } \\
\text { d'enseignement : le principe alphabétique et le } \\
\text { fonctionnement de la langue française sont-ils } \\
\text { expliqués? }\end{array}$ \\
\hline Q- 4 & $\begin{array}{l}\text { Les consonnes et les voyelles sont- } \\
\text { elles classées séparément au moins } \\
\text { une fois, de sorte que l'élève sache } \\
\text { distinguer entre ces deux catégories } \\
\text { de lettres? }\end{array}$ & \\
\hline Q- 3 & $\begin{array}{l}\text { Dans l'ensemble des séquences } \\
\text { d'apprentissage de l'alphabet, toutes } \\
\text { les lettres sont-elles présentées? }\end{array}$ & $\begin{array}{l}\text { Evaluer le degré de complétude des séquences } \\
\text { d'enseignement pour l'apprentissage de } \\
\text { l'alphabet : toutes les lettres sont- elles étudiées? } \\
\text { La richesse et la variété des combinaisons } \\
\text { syllabiques est-elle suffisantes? }\end{array}$ \\
\hline Q- 6 & $\begin{array}{l}\text { Toutes les combinaisons syllabiques } \\
\text { sont-elles présentées? }\end{array}$ & \\
\hline
\end{tabular}




\begin{tabular}{|c|c|c|}
\hline Q- 5 & $\begin{array}{l}\text { Au vu de l'ordre de présentation des } \\
\text { lettres, le critère de régularité } \\
\text { grapho-phonémique est-il pris en } \\
\text { compte (principe de progression } \\
\text { rationnelle)? }\end{array}$ & $\begin{array}{l}\text { Apprécier la progressivité des contenus } \\
\text { d'enseignement : vont- ils du plus simple au plus } \\
\text { complexe? }\end{array}$ \\
\hline Q- 7 & $\begin{array}{l}\text { La complexité des structures } \\
\text { syllabiques est-elle progressive? }\end{array}$ & \\
\hline Q- 8 & L'écrit est-il suffisamment abordé? & Evaluer la part consacrée à l'activité d'écriture. \\
\hline Q- 9 & $\begin{array}{l}\text { Les textes présentés (desquels sont } \\
\text { extraits les mots et syllabes étudiés) } \\
\text { sont-ils adaptés à l'environnement } \\
\text { cognitif et au niveau des élèves? }\end{array}$ & $\begin{array}{l}\text { Mesurer l'adaptation du vocabulaire utilisé dans } \\
\text { le manuel avec l'environnement cognitif et les } \\
\text { capacités de compréhension des élèves : les mots } \\
\text { présentés sont-ils en harmonie avec le niveau des } \\
\text { élèves? }\end{array}$ \\
\hline Q- 10 & $\begin{array}{l}\text { Les } \text { exercices interviennent-ils sur } \\
\text { des éléments présentés dans les } \\
\text { leçons? }\end{array}$ & $\begin{array}{l}\text { Estimer la concordance des activités proposées } \\
\text { avec l'enseignement: les exercices font-ils appel } \\
\text { à des notions (phonème ou graphème) qui n'ont } \\
\text { pas été apprises auparavant? Ou, au contraire, } \\
\text { portent-ils sur des éléments « étudiés? }\end{array}$ \\
\hline
\end{tabular}

Les réponses à ces questions permettront de cerner le processus cognitif sollicité et d'estimer son opportunité à la lumière des sciences cognitives.

Quant au manuel scolaire de référence, il est intitulé "Mon premier livre de français", comporte 125 pages et est composé de quatre (4) projets dont chacun comprend trois (3) séquences subdivisées en trois (3) activités :

Tableau II : Subdivision de l'activité pédagogique

\begin{tabular}{|l|l|l|}
\hline \multicolumn{1}{|c|}{ Projets } & \multicolumn{1}{|c|}{ Séquences } & \multicolumn{1}{c|}{ Activités } \\
\hline \multirow{2}{*}{$\begin{array}{l}\text { Réaliser un imagier thématique } \\
\text { sur l'école pour le présenter au } \\
\text { concours inter-écoles }\end{array}$} & S1: ma nouvelle école & $\begin{array}{l}\text { Oral - } \\
\text { Lecture - Ecriture }\end{array}$ \\
\cline { 2 - 3 } & S2: mes camarades de classe & $\begin{array}{l}\text { Oral - } \\
\text { Lecture - Ecriture }\end{array}$ \\
\cline { 2 - 3 } & S3: mon métier d'élève & $\begin{array}{l}\text { Oral - } \\
\text { Lecture - Ecriture }\end{array}$ \\
\hline \multirow{2}{*}{$\begin{array}{l}\text { Confectionner une affiche } \\
\text { illustrant des consignes de } \\
\text { sécurité routière pour la } \\
\text { présenter à une autre classe }\end{array}$} & S1: je suis piéton & $\begin{array}{l}\text { Oral - } \\
\text { Lecture - Ecriture }\end{array}$ \\
\cline { 2 - 3 } & S2 : je suis passager & $\begin{array}{l}\text { Oral - Lecture - } \\
\text { Ecriture }\end{array}$ \\
\cline { 2 - 3 } & $\begin{array}{l}\text { S3 : } \\
\text { je respecte le code de la route }\end{array}$ & $\begin{array}{l}\text { Oral - } \\
\text { Lecture - Ecriture }\end{array}$ \\
\hline
\end{tabular}




\begin{tabular}{|l|l|l|}
\hline \multirow{4}{*}{$\begin{array}{l}\text { Réaliser la fiche technique d'un } \\
\text { arbre fruitier pour la présenter à } \\
\text { l'exposition de la journée } \\
\text { mondiale de l'arbre, le } 21 \text { mars. }\end{array}$} & S1 : le coin vert & $\begin{array}{l}\text { Oral - } \\
\text { Lecture - Ecriture }\end{array}$ \\
\cline { 2 - 3 } & S2: Au marché & $\begin{array}{l}\text { Oral - } \\
\text { Lecture - Ecriture }\end{array}$ \\
\cline { 2 - 3 } & S3 : la fête de l'arbre & $\begin{array}{l}\text { Oral - } \\
\text { Lecture - Ecriture }\end{array}$ \\
\hline \multirow{2}{*}{$\begin{array}{l}\text { Confectionner un dépliant illustré } \\
\text { sur la préservation de } \\
\text { l'environnement pour l'afficher } \\
\text { dans la classe. }\end{array}$} & S1:j'économise l'eau & $\begin{array}{l}\text { Oral - } \\
\text { Lecture - Ecriture }\end{array}$ \\
\cline { 2 - 3 } & S2'économise l'électricité & $\begin{array}{l}\text { Oral - } \\
\text { Lecture - Ecriture }\end{array}$ \\
\cline { 2 - 3 } & S3:je protège mon & $\begin{array}{l}\text { Oral - } \\
\text { Lecture - Ecriture }\end{array}$ \\
\hline
\end{tabular}

Chaque page du manuel est analysée ; tous les éléments de son contenu sont évalués en fonction des critères de la grille organisée selon l'échelle de Likert. Les conclusions pour chaque question sont reportées sur le tableau suivi d'une analyse détaillée.

\section{Analyse du manuel de français : examen des données}

\section{- Données A}

\begin{tabular}{|l|c|c|c|c|}
\hline Question No 1 & $\begin{array}{c}\text { Tout à } \\
\text { fait }\end{array}$ & Plutôt & $\begin{array}{c}\text { Plutôt } \\
\text { pas }\end{array}$ & $\begin{array}{c}\text { Pas du } \\
\text { tout }\end{array}$ \\
\hline $\begin{array}{l}\text { Pour l'enseignement de l'alphabet, est-ce la méthode } \\
\text { syllabique, dite alphabétique ou traditionnelle qui est } \\
\text { utilisée? }\end{array}$ & & & & \\
\hline
\end{tabular}

Les séquences d'apprentissages sont divisées en trois activités progressives : l'oral, la lecture et l'écriture.

La première est l'oral : l'élève est invité à écouter des dialogues de bande dessinée qui sont lus par l'enseignant, puis à répéter certains mots ou phrases courtes qui apparaissent dans ces dialogues. Le but de cette activité est de développer le lexique des élèves.

La deuxième activité est la lecture : l'élève est invité à lire une bande dessinée, puis à lire un texte court en rapport avec celle-ci et duquel sont extraits quelques mots décomposés en lettres qu'il doit assembler par syllabes avant que des exercices de discrimination visuelle lui soient proposés.

Troisième et dernière activité est l'écriture : l'élève apprend à écrire les lettres étudiées dans les différentes graphies (scripte, cursive, minuscule et majuscule).

Dans le guide pédagogique destiné aux enseignants, ces différentes parties de l'acte pédagogique sont ainsi désignées : l'oral/réception, l'oral/production, l'écrit/réception et l'écrit/production (ONPS, 2008:2); et l'acte lui-même ainsi décrit (idem) : «Tu vas 
d'abord écouter des énoncés et apprendre des mots nouveaux. Ensuite, tu liras de jolis textes et tu écriras des petits messages ".

Chaque séquence d'apprentissage se termine par une situation d'intégration qui propose une activité en rapport avec le projet, et une comptine.

Concernant la méthode adoptée par le manuel pour l'enseignement de la lecture, le programme officiel fait référence aux deux processus cognitifs de reconnaissance (niveau bas) et d'interprétation (haut niveau), mais ne donne pas de précision quant à la méthode à adopter : "la mise en place de la lecture articulera décodage et compréhension dans une pédagogie de construction du sens par hypothèses et anticipation. L'élève sera mis directement en contact avec des textes écrits " (ONPS, $2011: 10$ ).

Le guide pédagogique palie cette carence en précisant qu'il s'agit de la "méthode synthétique de lecture, rapide, claire et motivante » (ONPS, 2008 :11), en ajoutant que dans le manuel, "plusieurs activités de lecture sont proposées [...] elles permettent par la discrimination visuelle de se familiariser avec les différentes graphies pour passer du son à la lettre puis au mot et à la phrase » (ONPS, $2008: 11$ 12).

Malgré l'approximation des orientations de ce guide, l'analyse du contenu du manuel révèle l'utilisation de la méthode mixte. Dans la pratique, cette méthode fait appel au lexique phonologique de l'apprenant.

Or, cela est possible dans l'enseignement de la lecture en langue maternelle ou seconde, puisque l'élève, avant même l'apprentissage de la lecture, dispose d'une quantité plus ou moins considérable de connaissances orales (dictionnaire phonologique); il est capable de nommer les objets qui lui sont présentés, et même de construire des phrases pour s'exprimer. Ce qui n'est pas le cas dans l'enseignement d'une langue étrangère, en raison de la pauvreté ou parfois même de l'inexistence de connaissances orales préalables chez l'apprenant.

Le fait que des activités orales soient proposées au début des séquences, permet en effet de développer le lexique des élèves, et la quantité et la qualité du vocabulaire qu'un enfant possède avant l'apprentissage de la lecture est décisive. En effet, selon Bentolila $(2006: 3)^{5}$ :

Certes, les relations entre lettres de l'alphabet et sons sont, pour certaines, complexes et parfois irrégulières, certes certaines structures grammaticales peuvent être spécifiques à l'oral, certes certains mots seront plus fréquemment utilisés à l'écrit, mais le cœur du système qui fonde la langue orale est bien celui qui permet à la langue écrite de fonctionner.

Mais la méthode préconisée pour l'apprentissage de la lecture en français langue étrangère ne prend pas en considération les difficultés propres à l'apprentissage d'une langue étrangère, ni les résultats récents des recherches sur l'apprentissage de la lecture en sciences cognitives évoqués plus haut.

\section{- Données B}

\begin{tabular}{|l|c|c|c|c|}
\hline \multicolumn{1}{|c|}{ Question $\mathrm{N}^{\mathrm{O}} 2$} & $\begin{array}{c}\text { Tout à } \\
\text { fait }\end{array}$ & Plutôt & $\begin{array}{c}\text { Plutôt } \\
\text { pas }\end{array}$ & $\begin{array}{c}\text { Pas du } \\
\text { tout }\end{array}$ \\
\hline $\begin{array}{l}\text { L'alphabet complet et en ordre est-il exposé de manière } \\
\text { pertinente à l'élève, de telle sorte qu'il puisse connaitre l'ordre } \\
\text { alphabétique? }\end{array}$ & & & & \\
\hline
\end{tabular}


C'est à la première séquence du quatrième projet, page 85 , qu'un encadré de cinq $(5) \mathrm{cm}$ sur trois (3), au milieu d'une page d'activités, présente l'alphabet complet et en ordre pour la première fois. Il précède un exercice dont la consigne est de ranger un ensemble de mots par ordre alphabétique. C'est aussi la première fois qu'il est question d'ordre alphabétique dans ce manuel.

Ce même encadré est repris quelques pages plus loin, page 93, dans le même contexte. Ce sont là les deux seules fois où le manuel scolaire évoque l'ordre alphabétique, et expose les lettres de l'alphabet au complet et en ordre.

Le fait que l'alphabet complet et en ordre ne soit pas présenté dans le manuel sinon de manière tardive, et uniquement dans le cadre d'une activité isolée, ne favorise pas l'acquisition de l'ordre alphabétique pourtant indispensable à la maitrise des «processus de bas niveau». Or, au niveau cognitif, cette "phase alphabétique » est essentielle à l'identification des mots et à l'automatisation requise pour la maîtrise d'une langue, et $a$ fortiori une langue étrangère.

\section{- Données C}

\begin{tabular}{|c|c|c|c|c|}
\hline \multicolumn{1}{|c|}{ Question No 3 } & $\begin{array}{c}\text { Tout à } \\
\text { fait }\end{array}$ & Plutôt & $\begin{array}{c}\text { Plutôt } \\
\text { pas }\end{array}$ & $\begin{array}{c}\text { Pas du } \\
\text { tout }\end{array}$ \\
\hline $\begin{array}{l}\text { Dans l'ensemble d'apprentissage des de séquences l'alphabet, } \\
\text { toutes les présentées ? lettres sont-elles présentées? }\end{array}$ & & & $\times$ & \\
\hline
\end{tabular}

Le manuel est divisé en quatre projets et chaque projet comporte trois (3) séquences. Les lettres de l'alphabet sont segmentées en huit ensembles de trois à quatre unités. Dans chaque séquence, un ensemble de lettres est présenté.

Dans toutes les séquences d'apprentissage de l'alphabet, aucune mention n'est faite de deux lettres: Y et W. Cette omission, qui n'a pas été corrigée dans les éditions suivantes, est pénalisante pour l'élève. En effet, si l'enseignant ne remédie pas à cette lacune, l'élève sera dans l'incapacité de lire ou de comprendre les mots contenant ces lettres; son apprentissage sera incomplet, et son aptitude à suivre la suite des apprentissages s'en trouvera compromise.

\section{- Données D}

\begin{tabular}{|c|c|c|c|c|}
\hline Question $\mathbf{N}^{\mathbf{0}} 4$ & $\begin{array}{c}\text { Tout à } \\
\text { fait }\end{array}$ & Plutôt & $\begin{array}{c}\text { Plutôt } \\
\text { pas }\end{array}$ & $\begin{array}{c}\text { Pas du } \\
\text { tout }\end{array}$ \\
\hline $\begin{array}{l}\text { Les consonnes et les voyelles sont-elles classées séparément au } \\
\text { moins une fois, de sorte que l'élève sache distinguer entre ces } \\
\text { deux catégories de lettres? }\end{array}$ & & & & \\
\hline
\end{tabular}

À aucun moment, la distinction entre voyelles et consonnes n'est indiquée ou enseignée à l'élève. Le principe alphabétique ainsi enseigné, et donc acquis de façon réductrice, prive l'élève de la visibilité sur le fonctionnement de la langue. Ainsi, il n'apprendra pas, par exemple, que les voyelles peuvent modifier la prononciation de consonnes; le cas contraire est plus rare en français parce que les voyelles sont en général régulières. 
D'ailleurs, Dehaene S. et al. (2012: 70) préconisent le principe de l'enseignement explicite du code alphabétique, "en montrant comment une même consonne, combinée à différentes voyelles en modifie la prononciation ('la', 'le', 'li', 'lo', 'lu'), et inversement ('la', 'ra', ' $m a$ ', etc.) ».

\section{- Données E}

\begin{tabular}{|l|c|c|c|c|}
\hline Question $\mathbf{N}^{\circ} 5$ & $\begin{array}{c}\text { Tout à } \\
\text { fait }\end{array}$ & Plutôt & $\begin{array}{c}\text { Plutôt } \\
\text { pas }\end{array}$ & $\begin{array}{c}\text { Pas du } \\
\text { tout }\end{array}$ \\
\hline $\begin{array}{l}\mathrm{Au} \mathrm{vu} \mathrm{de} \mathrm{l'ordre} \mathrm{de} \mathrm{présentation} \mathrm{des} \mathrm{lettres,} \mathrm{le} \mathrm{critère} \mathrm{de} \\
\text { régularité grapho- phonémique est-il pris en compte (principe } \\
\text { de progression rationnelle)? }\end{array}$ & & & & \\
\hline
\end{tabular}

Dans le manuel, les lettres de l'alphabet sont présentées dans cet ordre :

\begin{tabular}{|c|c|c|c|c|c|c|}
\hline Séquence $\mathrm{n}^{0} 1$ & A & I & M & $\mathrm{N}$ & & \\
\hline Séquence $\mathrm{n}^{0} 2$ & E & É & $\mathrm{T}$ & $\mathrm{D}$ & & \\
\hline Séquence $n^{0} 3$ & $\mathrm{~L}$ & 0 & $\mathrm{R}$ & & & \\
\hline Séquence $\mathrm{n}^{\mathrm{O}} 4$ & $\mathrm{U}$ & OU & $\mathrm{P}$ & B & & \\
\hline Séquence $\mathrm{n}^{0} 5$ & $\mathrm{~F}$ & V & OI & & & \\
\hline Séquence $n^{0} 6$ & S & $\mathrm{Z}$ & $\mathrm{X}$ & & & \\
\hline Séquence $\mathrm{n}^{0} 7$ & C & K & $Q$ & G & $\mathrm{AM} / \mathrm{AN}$ & EM/EN \\
\hline Séquence $n^{0} 8$ & $\mathrm{H}$ & $\mathrm{J}$ & $\mathrm{CH}$ & ON/OM & & \\
\hline Séquence $\mathrm{n}^{0} 9$ & $\frac{G}{\text { prononcé ' } G \text { ' }}$ & $\begin{array}{c}\text { G } \\
\text { Prononcé 'J' }\end{array}$ & & & & \\
\hline Séquence $\mathrm{n}^{\mathrm{O}} 10$ & AI & IN & EU & & & \\
\hline Séquence $\mathrm{n}^{0} 11$ & IEL & EU & UN & & & \\
\hline Séquence $\mathrm{n}^{\mathrm{O}} 12$ & UI & GN & & & & \\
\hline
\end{tabular}

L'analyse de ce tableau nous permet de relever un certain déséquilibre dans la quantité de lettres présentées à chaque séquence: par exemple, quatre dans l'une (séquence $n^{\circ} 1$ ), quatre lettres et quatre combinaisons syllabiques dans l'autre (séquence $n^{\circ} 7$ ).

On observe aussi que les lettres sont confondues avec les combinaisons syllabiques, ce qui ne permet pas à l'élève de les distinguer clairement.

Aussi, nous pouvons en déduire que le principe de progression rationnelle n'est pas respecté : l'ordre dans lequel sont présentées les lettres dans le manuel ne prend pas en considération le critère de régularité. 
En effet, l'analyse de la langue française révèle que concernant les lettres, celles dont les correspondances graphèmes-phonèmes sont les plus régulières (c'est-à-dire celles qui se prononcent toujours de la même manière) sont les voyelles (elles connaissent de très rares irrégularités (Dehaene et al., 2011). C'est la raison pour laquelle non seulement elles doivent être étudiées en tant que telles mais elles doivent l'être en premier. Ce qui n'est pas le cas dans ce manuel.

Autre exemple : le J, qui est un graphème régulier, n'est abordé qu'à la $8^{\text {ème }}$ séquence, alors que des graphèmes irréguliers $(\mathrm{C}-\mathrm{S}-\mathrm{T}-$...) sont abordés dans les séquences qui la précèdent.

On observe que l'ordre de présentation des lettres ne tient pas compte des paramètres linguistiques: les lettres de l'alphabet sont présentées de manière aléatoire; il ne semble s'en dégager aucune logique particulière.

\section{- Données F}

\begin{tabular}{|c|c|c|c|c|}
\hline Question No 6 & $\begin{array}{c}\text { Tout à } \\
\text { fait }\end{array}$ & Plutôt & $\begin{array}{c}\text { Plutôt } \\
\text { pas }\end{array}$ & $\begin{array}{c}\text { Pas du } \\
\text { tout }\end{array}$ \\
\hline $\begin{array}{l}\text { Toutes les combinaisons syllabiques sont-elles } \\
\text { présentées? }\end{array}$ & & & $\times$ & \\
\hline
\end{tabular}

11 L'étude attentive et détaillée de l'ensemble des séquences d'apprentissage des lettres de l'alphabet et des combinaisons syllabiques révèle que :

- toutes les combinaisons syllabiques ne sont pas présentées : dans certaines séquences, un graphème n'est introduit que dans deux ou trois combinaisons; toutes les voyelles n'ayant pas été étudiées en premier, les combinaisons consonnes-voyelles sont restreintes ; mais ce n'est pas la raison de la pauvreté des combinaisons syllabiques, parce que même une fois étudiées, les voyelles ne sont pas nécessairement combinées avec les consonnes à étudier ;

- la mobilité des graphèmes (déplacement de lettres et changement de prononciation) n'est prise en compte que dans deux séquences d'apprentissage sur dix (séquence 3, séquence 6).

- dans la séquence 7 , toutes les combinaisons syllabiques comprenant le $C$, illustrent le $C$ dur (ca - co - cou - cra - etc) ; aucune combinaison syllabique ne comprend le $\mathrm{C}$ doux (ci - ce etc.).

12 L'insuffisance des combinaisons syllabiques présentées est pénalisante pour l'élève. Sans l'intervention de l'enseignant, son aptitude à suivre la suite des apprentissages sera compromise.

\section{- Données G}

\begin{tabular}{|c|c|c|c|c|}
\hline Question $N^{\circ} 7$ & $\begin{array}{c}\text { Tout à } \\
\text { fait }\end{array}$ & Plutôt & $\begin{array}{c}\text { Plutôt } \\
\text { pas }\end{array}$ & $\begin{array}{c}\text { Pas du } \\
\text { tout }\end{array}$ \\
\hline $\begin{array}{l}\text { La complexité des structures syllabiques est-elle } \\
\text { progressive? }\end{array}$ & & & $\times$ & \\
\hline
\end{tabular}


Dans les deux premières séquences, les combinaisons syllabiques présentées sont toutes des structures consonne-voyelle $(\mathrm{CV})$. À partir de la troisième séquence, des combinaisons de structure voyelle-consonne (VC) sont introduites, et à partir de la quatrième séquence, on rencontre des combinaisons de type consonne-voyelle- voyelle (CVV), consonne- consonne-voyelle (CCV) et consonne-consonne-voyelle-voyelle (CCVV).

En voici une illustration : pa - po / pou / pli - plu - pra - pri - pro - pru - plou - prou/ - etc. (p. 35).

A partir de la quatrième séquence aucune progression dans la présentation des syllabes n'est observée.

\section{- Données H}

\begin{tabular}{|c|c|c|c|c|}
\hline Question $N^{\circ} 8$ & Tout à fait & Plutôt & Plutôt pas & Pas du tout \\
\hline L'écrit est-il suffisamment abordé ? & & & $\times$ & \\
\hline
\end{tabular}

Chaque séquence d'apprentissage, jusqu'à la huitième, comporte une page consacrée à l'écriture. Au-delà, le manuel ne propose aucune activité d'écriture.

Or, de nombreuses recherches ont démontré que l'enfant, par l'exploitation tactile des lettres et le geste d'écriture, améliore sa lecture (Gentaz, É., Colé, P., Bara, F., Bara, F. 2003 ; Gentaz, É., Colé, P., Sprenger-Charolles. 2004) ; car, " en ajoutant un code moteur au répertoire mental des lettres, ces activités facilitent la mémoire des correspondances graphèmesphonèmes et réduisent les ressemblances entre les lettres » (Dehaene et al.., 2011 : 86).

\section{- Données I}

\begin{tabular}{|l|c|c|c|c|}
\hline \multicolumn{1}{|c|}{ Question $\mathbf{N}^{\mathbf{0}}$ 9 } & $\begin{array}{c}\text { Tout à } \\
\text { fait }\end{array}$ & Plutôt & $\begin{array}{c}\text { Plutôt } \\
\text { pas }\end{array}$ & $\begin{array}{c}\text { Pas du } \\
\text { tout }\end{array}$ \\
\hline $\begin{array}{l}\text { Les textes présentés (desquels sont extraits les mots et syllabes } \\
\text { étudiés) sont-ils adaptés à l'environnement cognitif et au } \\
\text { niveau des élèves? }\end{array}$ & & & & \\
\hline
\end{tabular}

Pour estimer le degré d'adaptation du contenu du manuel au niveau des apprenants et à leur environnement cognitif, nous avons évalué le degré de difficulté (lisibilité/ intelligibilité) des textes qui leur sont proposés. Pour ce faire, nous avons d'abord opéré une évaluation quantitative, c'est-à-dire que nous avons évalué la longueur des textes en question, ce qui est un précieux indice sur la lisibilité syntaxique, puis, nous avons étudié le contenu sémantique des textes (lisibilité lexicale).

À propos de la lisibilité syntaxique, les textes présentés aux apprenants sont d'une quinzaine de mots dans les premières séquences et jusqu'à une quarantaine dans les dernières.

Quant à la lisibilité lexicale, le choix du vocabulaire dans certains textes a particulièrement retenu notre attention : en raison de leur difficulté, nous avons jugé ces textes inadaptés parce que leur vocabulaire est peu courant, sa régularité grapho- 
phonémique est très faible et il ne fait pas partie de l'environnement cognitif des apprenants ou du moins de la majorité d'entre eux.

En voici quelques exemples : comptine (P.19), Wagon (P.39), signe (P.51), vergers - greniers - champs (P.59), agrumes (P.75), timbale (P.85), hygiène (P.90), s'éteignent (P.91), cygne (P. 99), etc.

De plus, les textes proposés dans le manuel présentent un volume lexical important, et englobent un large champ lexical. Or, comme le soulignent Dehaene et al.. (2011:48) :

Dans la première année d'école primaire, la lecture demande à l'enfant un immense effort d'attention. Déchiffrer les mots implique de passer en revue chacune des lettres dans le bon ordre, de la gauche vers la droite, sans en oublier une seule, tout en se souvenant de leurs correspondances avec les phonèmes et en les assemblant en mémoire pour former un mot. Chaque mot est une énigme, un puzzle que l'enfant ne reconstitue qu'au prix de grands efforts.

Les textes du manuel requièrent de l'apprenant un grand effort au niveau cognitif, et cela constitue une entrave à l'apprentissage.

En effet :

un coût [cognitif] élevé du décodage surtout pendant la phase d'apprentissage peut gêner la réalisation des traitements de haut niveau concernant l'intégration textuelle. Inversement, des difficultés de compréhension dues, par exemple, à une méconnaissance du thème évoqué par le texte peuvent altérer le décodage. (Fayol, Gombert et al., 1992:42)

Notons également que la surcharge cognitive peut induire chez l'apprenant un sentiment d'impuissance qui mènera au rejet de la langue.

\section{- Données J}

\begin{tabular}{|c|c|c|c|c|}
\hline Question No 10 & $\begin{array}{c}\text { Tout à } \\
\text { fait }\end{array}$ & Plutôt & $\begin{array}{c}\text { Plutôt } \\
\text { pas }\end{array}$ & $\begin{array}{c}\text { Pas du } \\
\text { tout }\end{array}$ \\
\hline $\begin{array}{l}\text { Les exercices interviennent-ils sur des éléments } \\
\text { présentés dans les leçons? }\end{array}$ & & $\times$ & & \\
\hline
\end{tabular}

Après chaque séquence d'apprentissage des lettres de l'alphabet, diverses activités sont proposées. Dans ces exercices, sont souvent utilisés des graphèmes n'ayant pas été étudiés.

Par exemple, après la première séquence, où sont présentées les quatre lettres /A, I, M, $\mathrm{N} /$ et les combinaisons syllabiques /ma, mi, na, ni/, un exercice est proposé :

- compléter les vides avec la lettre qui manque (sans préciser laquelle) :

1. Une /oto / (au-dessous d'une image représentant une moto) - P13

2. Un /id/ (au-dessous d'une image représentant un nid) - P13

Dans le premier mot, l'image facilite la réponse, puisque l'élève en disant ce qu'il voit donne la réponse correcte (le mot faisant partie de son lexique phonologique). Mais il ne s'agit pas d'une lecture réelle des deux syllabes qui forment ce mot - MO / TO puisque ce sont deux combinaisons syllabiques que l'élève n'a pas encore apprises et qu'il n'a même jamais rencontrées auparavant dans le manuel.

Le deuxième mot, "nid», est encore plus compliqué : d'abord parce que la probabilité qu'il fasse partie du lexique phonologique des élèves est faible, ensuite parce qu'il contient une lettre muette (D). Ajoutons que l'élève ne sait pas encore décoder les articles « un » et « une », puisqu'il n'a pas encore étudié ces graphèmes.

Un autre exemple : l'exercice de la page 53 : 


\begin{tabular}{|c|c|c|c|c|c|c|}
\hline \multicolumn{7}{|c|}{ Je complète les mots avec : l' - le - la - les: } \\
\hline ... bureau & - & ... eau & - & ... gaufrette & - & ... tableau \\
\hline ... rideaux & - & ... auto & - & ... roseau & - & ... Taureaux \\
\hline
\end{tabular}

Avant cet exercice, aucune leçon et ni même aucune mention des articles définis (masculin, féminin/ singulier, pluriel) n'ont été faites et le graphème «LES » n'a pas été étudié.

Le manuel propose ainsi dans chaque séquence des exercices qui requièrent des connaissances avant leur acquisition par l'apprenant. Ainsi, il est fait systématiquement appel au lexique oral de l'élève, à qui il est demandé de trouver ou de compléter, en langue étrangère, les noms des objets présentés, alors que ce même lexique est censé être un objet d'apprentissage.

Le manuel scolaire est reconnu comme l'un des facteurs les plus efficaces pour améliorer la qualité de l'enseignement, surtout dans les pays où le système éducatif manque de moyens (Seguin, 1989; Gerard \& Roegiers, 2003 2009), où les classes sont surchargées comme c'est le cas en Algérie. C'est pourquoi investir cet outil et travailler à parfaire la qualité de son contenu à la lumière des avancées scientifiques récentes, comme celles des sciences cognitives sollicitées ici, devrait être une priorité, notamment pour les apprentissages en classes primaires.

C'est dans cette perspective que cette modeste étude a mis au jour les carences du manuel scolaire de la première année de français en cycle primaire. Elle offre la possibilité de proposer des améliorations de ce manuel dans le respect des résultats actualisés de la psychologie cognitive.

\section{BIBLIOGRAPHIE}

ADEL, F., « l'élaboration des nouveaux programmes scolaires », in La Refonte de la pédagogie en Algérie, Bureau International de l'Education, UNESCO, Ministère de l'Education Nationale, Algérie, 2005, pp. 45-56.

BARA, F., GENTAZ, É., COLE, P., SPRENGER-CHAROLLES, L., « The visuo-haptic and haptic exploration of letters increases the kindergarten-children's understanding of the alphabetic principle », in Cognitive development, 19 (3), 2004, pp. 433-449.

BENTOLILA, A., « Apprendre à lire », 2006. Disponible sur le site :

$<$ http://www.bienlire.education.fr >

BONNEFOY, B., REY, A., « Automatisation de la connaissance des lettres chez l'apprenti lecteur », in L'année psychologique, vol. 108, n², 2008, pp. 187-206.

BULLETIN MENSUEL D'INFORMATION du Centre National de Documentation Pédagogique (C.N.D.P.), $\mathrm{n}^{\circ}$ 57, Alger, novembre 2003. 
DEHAENE, S., (dir.), Apprendre à lire : Des sciences cognitives à la salle de classe, Odile Jacob, Paris, 2011.

DEHAENE, S., \& COHEN, L., "The unique role of the visual word form area in reading", in Trends in cognitive sciences, 15 (6), 2011, pp. 254-262.

DEHAENE, S., « Psychologie cognitive expérimentale : Cours : Les mécanismes cérébraux de la lecture » (document d'enseignement). Disponible sur le site :

< https://www.college-de-france.fr/media/stanislas-dehaene/UPL54166_18.pdf >

DEHAENE, S., HURON, C. et SPRENGER-CHAROLLES, L, « Quelques grands principes de l'apprentissage de la lecture », 2012. Disponible sur le site :

< http://www.ac-grenoble.fr/ien.bourgoin2/IMG/pdf/

Quelques_grands_principes_de_l_apprentissage_de_la_lecture_et_progression_Dehaene.pdf >

DEMEUSE, M., Introduction aux théories et aux méthodes de la mesure en sciences psychologiques et en sciences de l'éducation, Editions de l'Université de Liège, 2004.

DEMONT, Élisabeth, GOMBERT, Jean-Émile, « L'apprentissage de la lecture : évolution des procédures et apprentissage implicite », in Enfance, (Vol. 56), 2004, p. 245-257.

FAYOL, M., GOMBERT, J-É., LECOCQ, P., SPRENGER-CHAROLLES, L., ZAGAR, D., Psychologie cognitive de la lecture. PUF, 1992.

FERHANI, F., F., « L'enseignement du français à la lumière de la réforme », in Le français aujourd'hui, $\mathrm{n}^{\circ}$ 154, 2006, pp. 11-18. Disponible sur [URL] :

< https://www.cairn.info/revue-le-francais-aujourd-hui-2006-3-page-11.htm >

GAONAC'H, D., « Lire dans une langue étrangère : approche cognitive », in Revue Française de Pédagogie, $\mathrm{n}^{\circ}$ 93, paris, INRP, 1990, pp. 75-100.

GAONAC'H, D., « Les stratégies attentionnelles dans l'utilisation d'une langue étrangère », dans Gaonac'h, D., (coord.), Acquisition et utilisation d'une langue étrangère, Paris, Hachette, 1990, pp. 41-49.

GAONAC'H, D., « La lecture en langue étrangère : un tour d'horizon d'une problématique de psychologie cognitive ", in Acquisition et interaction en langue étrangère, $\mathrm{n}^{\circ} 13,2000$, pp. 5-14.

GENTAZ, É. \& DESSUS, P. (dirs), Comprendre les apprentissages : sciences cognitives et éducation, Paris, Dunod, 2004

GENTAZ, E., COLE, P. \& BARA, F., « Evaluation d'entraînements multisensoriels de préparation à la lecture pour les enfants de grande section maternelle : une étude sur la contribution du système haptique manuel », in L'Année Psychologique, 104, 2003, pp. 561-584.

GERARD F. M., \& ROEGIERS X. et al., Des Manuels scolaires pour apprendre. Concevoir, évaluer, utiliser, De Boeck, 2003 ( $2^{\mathrm{e}}$ ed. 2009).

GERARD, F. M. \& ROEGIERS, X., Concevoir et évaluer des manuels scolaires, De Boeck Université, Bruxelles, 1993.

INRP, Lire et écrire à l'école primaire, état des recherches à l'INRP, ouvrage collectif, Institut national de recherche pédagogique, 1994.

JAMET, É., Lecture et réussite solaire, DUNOD, Paris, 1997.

JOULIA, D., La lecture en langue étrangère : approche cognitive et logiciels hypermédias, in Lenguaje, $\mathrm{n}^{\circ} 34,2006$, pp. 160184. 
LERAY, C., L'analyse de contenu : de la théorie à la pratique la méthode Morin-Chartier, Presses de l'Université du Québec, Québec, 2008.

ROCHE, M., « Apprentissage de la lecture : du décodage à la compréhension », Education, 2013. Disponible sur le site :

$<$ http://dumas.ccsd.cnrs.fr/dumas-00904427 >

SEGUIN, R., L'Elaboration des manuels scolaires. Guides pédagogiques, UNESCO, 1989. Disponible sur le site :

< http://unesdoc.unesco.org/images/0008/000869/086964F.pdf >

SPRENGER-CHAROLLES, L., « L'acquisition de la lecture en français : étude longitudinale de la première à la seconde année du primaire », in L'année psychologique, 94(4), 1994, pp. 553-574.

TAWIL, S., "Introduction aux enjeux et aux défis de la refonte pédagogique en Algérie », dans La refonte de la pédagogie en Algérie : défis et enjeux d'une société en mutation, Bureau International de l'Education, UNESCO, Ministère de l'éducation nationale, Algérie, 2005, pp. 33-44.

\section{Références du corpus}

ONPS, Mon premier livre de français, manuel pour la $3^{\text {ème }}$ année primaire, ONPS, Alger, 2008.

ONPS, Guide pédagogique, manuel scolaire de français $3^{\text {ème }}$ année primaire, ONPS, Alger, 2008.

ONPS, Programme de français de la $3^{\circ}$ année primaire, ONPS, Alger, 2008.

ONPS, Document d'accompagnement du programme de français de la $3^{\circ}$ et $4^{\circ}$ année primaire, ONPS, Alger, 2008 .

\section{ANNEXES}

Principe de l'apprentissage de la lecture (Dehaene et al.., 2011)

\begin{tabular}{|l|l|}
\hline & $\begin{array}{l}\text { P1a. Combinatoire des lettres (ou des } \\
\text { graphèmes). } \\
\text { Plphabétique. }\end{array}$ \\
\hline & $\begin{array}{l}\text { P1b. Mobilité des lettres (ou des graphèmes). } \\
\text { P1c. Correspondances spatio-temporelle. } \\
\text { P1d. Discrimination en miroir. }\end{array}$ \\
\hline P2. Principe de progression rationnelle. & $\begin{array}{l}\text { P2a. Régularité des relations graphèmes- } \\
\text { phonème. } \\
\text { P2b. Fréquence des graphèmes et des } \\
\text { phonèmes. } \\
\text { P2c. Facilité de prononciation des consonnes } \\
\text { isolées. } \\
\text { P2d. Complexité de la structure syllabique. } \\
\text { P2e. Inséparabilité des graphèmes } \\
\text { complexes. } \\
\text { P2f. Lettres muettes. } \\
\text { P2g. Fréquences des mots. } \\
\text { P2h. Rôle des morphèmes. }\end{array}$ \\
\hline
\end{tabular}


P3. Principe d'apprentissage actif associant lecture et écriture.

P4. Principe de transfert de l'explicite vers l'implicite.

\begin{tabular}{|l|l|}
\hline & $\begin{array}{l}\text { P5a. Concordance avec l'enseignement. } \\
\text { P5. Principe de choix rationnel des exemples et des } \\
\text { exercices. }\end{array}$ \\
& $\begin{array}{l}\text { P5c. Distinction entre le nom et le son des } \\
\text { lettres. } \\
\text { P5d. Variété des exemples et des exercices. }\end{array}$ \\
\hline
\end{tabular}

P6. Principe d'engagement actif, d'attention et de plaisir

P7. Principe d'adaptation au niveau de l'enfant

\section{NOTES}

1. Pour le contenu de la réforme voir Bulletin Mensuel d'Information du Centre National de Documentation Pédagogique (C.N.D.P.), n 57, Alger, novembre 2003.

2. Nous traduisons: «En 2000, en utilisant l'imagerie par résonance magnétique fonctionnelle (FMRI) sur des sujets normaux et chez deux patients présentant des lésions colossales, nous avons trouvé que la lecture est associée à l'activation d'un site précis et reproductible dans la région occipito-temporale ventrale gauche... Nous avons provisoirement appelé cette région aire de la forme visuelle des mots (VWFA)».

3. Ces points ont été traités par l'auteur dans un article intitulé « La lecture en langue étrangère : un tour d'horizon d'une problématique de psychologie cognitive", Acquisition et interaction en langue étrangère [En ligne], 13 | 2000, mis en ligne le 13 décembre 2005. URL: http:// aile.revues.org/970

4. «Il [Likert] soumet directement aux sujets dont il souhaite mesurer l'attitude, des propositions à juger selon une échelle prédéterminée, du type : Tout à fait d'accord - D'accord - Sans avis - En désaccord - Tout à fait en désaccord. Le nombre des modalités possibles peut varier (généralement de 3 à 7), mais correspond le plus souvent à un nombre impair de manière à ménager une modalité relativement neutre ou non polarisée au centre de l'échelle. Le nombre de modalités proposées est fonction à la fois de la précision souhaitée, mais aussi de la capacité présumée des sujets à effectuer des discriminations fines, un trop grand nombre de catégories n'entraînant pas nécessairement une augmentation de la précision", cf. Marc Demeuse, "Echelles de Likert ou méthodes de classements additionnés ", dans Demeuse Marc, Introduction aux théories et aux méthodes de la mesure en sciences psychologiques et en sciences de l'éducation, Editions de l'Université de Liège, 2004, p. 213. Mis en ligne en janvier 2008. Disponible sur le site : < http://iredu.u-bourgogne.fr/images/stories/Documents/Cours_disponibles/Demeuse/Cours/ p5.3.pdf >

5. Cf. Extrait de l'article intitulé « Apprendre à lire », mars 2006. Consultable sur le site : < http:// www.bienlire.education.fr > 


\section{RÉSUMÉS}

Cette étude porte sur l'enseignement-apprentissage de la lecture en langue française tel qu'élaboré dans le manuel scolaire consacré à la première année d'apprentissage du français langue étrangère. Il s'agit de l'unique manuel scolaire pour ce niveau et pour cette matière, en Algérie, depuis 2008. L'article confronte le contenu de ce support pédagogique aux résultats récents des recherches sur l'apprentissage de la lecture en sciences cognitives et en psychologie. Nous nous appuyons sur une grille d'analyse qui comporte dix (10) questions dont les réponses sont réparties sur une échelle de type Likert. Nous concluons que les grands principes et recommandations issus de ces recherches ne sont pas pris en considération pour l'enseignement de la lecture dans ce manuel.

This study focuses on the teaching and learning of reading in French as developed in the school textbook of the first year of learning French as a foreign language. It is the only textbook in Algeria for that level and for that discipline, since 2008. This paper compares the contents of this teaching support to recent research findings on learning to read in cognitive sciences and psychology. Relying on an analytical grid comprising ten (10) questions whose answers are distributed on a Likert -type scale. We conclude that the main principles and recommendations resulting from researches in psychology and cognitive sciences are not taken into account for the teaching of reading in this textbook.

\section{INDEX}

Mots-clés : psychologie cognitive, enseignement-apprentissage de la lecture, français langue étrangère, manuels scolaires, bas niveau, haut niveau

Keywords : cognitive psychology, teaching and learning to read, French language, school textbooks, low level, high level

\section{AUTEURS}

\section{ATFA MEMAÏ}

Laboratoire d'Analyse des Processus Sociaux et Institutionnels (LAPSI) Université Constantine 2 Abdelhamid Mehri Constantine - Algérie

\section{ABLA ROUAG}

Laboratoire d'Analyse des Processus Sociaux et Institutionnels (LAPSI) Université Constantine 2 Abdelhamid Mehri Constantine - Algérie 